\title{
Manifestationen selbstverletzenden Verhaltens in der HNO
}

S. Rudolph ${ }^{1}$, U. Schu ${ }^{2}$ J.A. Werner ${ }^{1}$, B.J. Folz ${ }^{1}$

1 Klinik für Hals-Nasen-Ohren-Heilkunde (Direktor: Prof. J.A. Werner) der Philipps-Universität Marburg

2 Klinik für Psychiatrie und Psychotherapie (Direktor: Prof. J.-Ch. Krieg) der Philipps-Universität Marburg

www.hno-marburg.de

\section{Einleitung}

Selbstverletzendes Verhalten kann im Rahmen verschiedener psychiatrischer Erkrankungen auftreten, wobei von den Betroffenen berichtet wird, dass die Verletzung dazu dient, innere Spannungen abzubauen. Häufig manifestiert sich selbstverletzendes Verhalten durch Manipulationen an den Extremitäten, insbesondere in Form von Schnittverletzungen an den Unterarmen. Zum KopfHalsbereich finden sich nur wenige Mitteilungen über Manifestationen eines solchen Verhaltens. Hier sollen 2 Fälle vorstellt werden, die exemplarisch für Manifestationen selbstverletzenden Verhaltens im Kopf-Hals-Bereich sind.

\section{Kasuistik 1}

Im März 1999 wurde eine 18-jährige Patientin mit Schmerzen über der linken Kieferhöhle erstmals vorgestellt. In der hno-ärztlichen Untersuchung fand sich ein Reissnagel in der linken Nasenhaupthöhle, Eine Röntgen-Untersuchung der Nasennebenhöhlen schloss weitere FK aus. In der durchgeführten Panendoskopie konnten keine weiteren Fremdkörper im Bereich der oberen Luft- und Speisewege identifiziert werden. Der Fremdkörper konnte problemlos entfernt werden. Im Zeitraum von März 1999 bis Dezember 2001 erfolgten insgesamt 66 weitere Vorstellungen mit inkorporierten Fremdkörpern in unterschiedlichen Intervallen. Dabei fanden sich insgesamt 68 Fremdkörper in der Nasenhaupthöhle, im Gehörgang, im Nasenrachenraum, im Oropharynx und Magen. Dabei handelte es sich unter anderem um: Reissnägel, Rasierklingen, Glasscherben, Plastikstücke, Metallschrauben, Sekundenkleber, Dosenverschlüsse, Antennenteilstücke, Sicherheitsnadelteilstücke, Batterien, Knöpfe, CD-Bruchstücke, Messerspitzen und Steine. Die Patientin stellte sich zum Teil mit multiplen Fremdkörpern vor, die sich auch an unterschiedlichen Lokalisationen fanden. In 9 Fällen fanden sich zweite Fremdkörper in anderen Körperteilen, dreimal in der Vagina und sechsmal in verschiedenen Teilen des Gastro-Intestinaltraktes. Aus der Anamnese der Patientin sind eine Borderline-Persönlichkeitstörung in Zusammenhang mit einer familiären Missbrauchssymptomatik, sowie eine hereditäre Epilepsie und eine mentale Retardierung bekannt. Im Alter von sieben Jahren war die Patientin mit einer Entwicklungsverzögerung aufgefallen und lebte seither vor allem in kinder- und jugendpsychiatrischen Einrichtungen. Die Patientin fiel dort durch ein emotional instabiles Verhalten mit abhängigen Zügen, Somatisierung, selbstverletzenden Tendenzen, komplex-partiellen und später psychogenen Anfällen auf. Seit ihrem 16. Lebensjahr kam es zunehmend zu selbstverletzenden Tendenzen, vor allem in Form von Inkorporationen in Vagina, Nase, Ohren, After und Augen
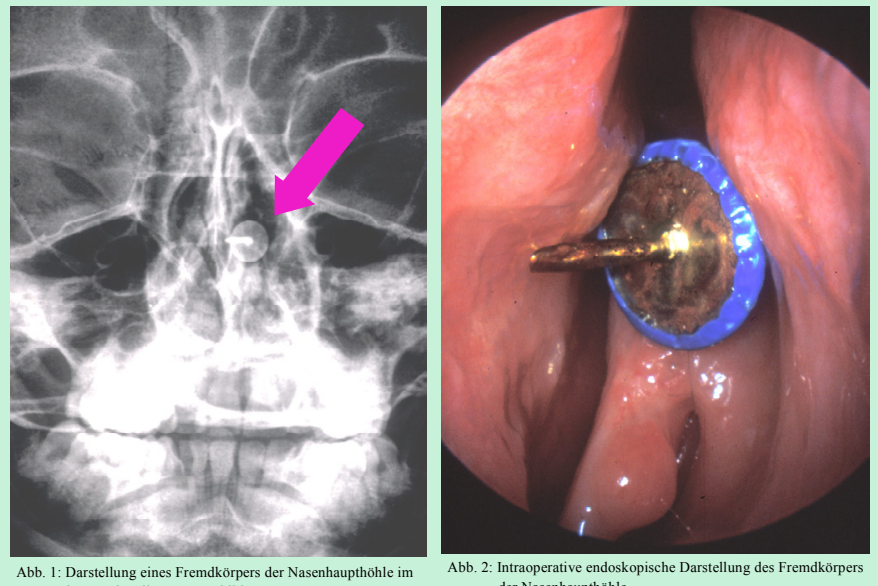

Abb. 1: Darstellung eines Fremdkörper

Intraoperative endos
der Nasenhaupthöhle
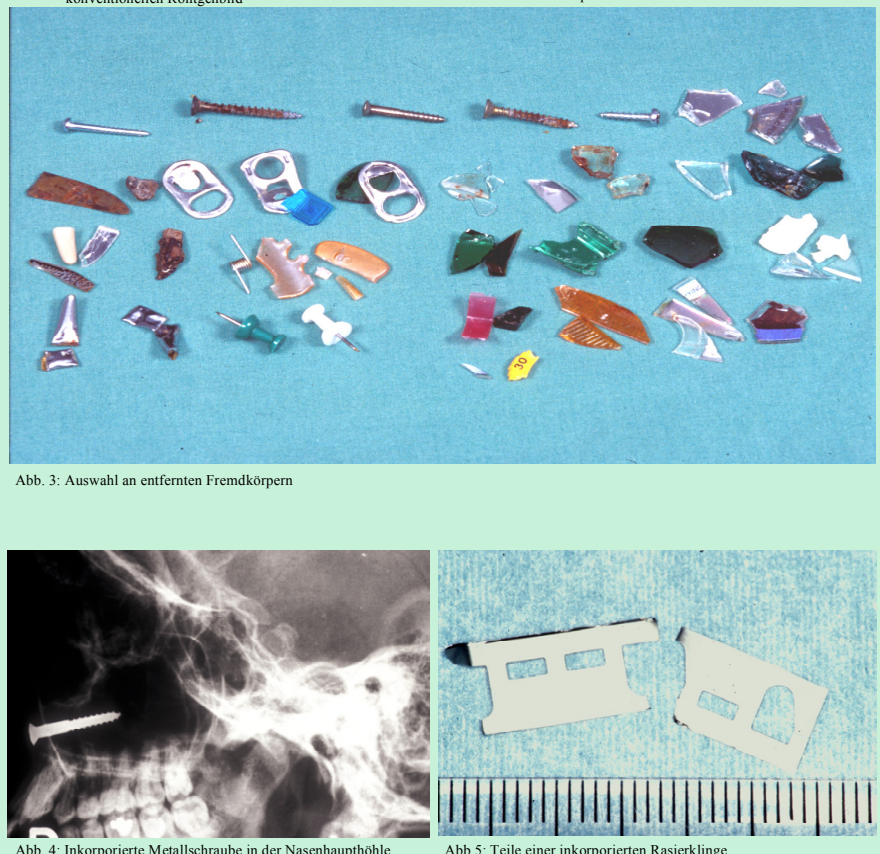

Es fand sich eine hohe Übereinstimmung zwischen den Angaben der Patientin und den gefundenen Fremdkörpern, lediglich sechsmal konnte trotz gegenteiliger Aussagen kein Fremdkörper identifiziert werden. Zur Sicherung der Diagnose und Entfernung der Fremdkörper waren insgesamt sechs stationäre Aufenthalte und 23 Intubations- oder Maskennarkosen, neben den zahlreichen ambulanten Vorstellungen und Entfernungen ohne Sedierung in Lokalanästhesie notwendig. Die Behandlung fand stets in Zusammenarbeit mit den behandelnden und konsiliarisch tätigen Psychiatern statt. Direkt nach erfolgter Inkorporation wurden die Hände mit Handschuhen versorgt, die ein weiteres Greifen und Inkorporieren von Gegenständen [Abb.6] unmöglich machten. Eine Einweisung in eine geschlossene Abteilung der Psychiatrie zur akuten Intervention wurde zu keinem Zeitpunkt empfohlen, da dies die akute psychiatrische Situation der Patientin verschlechtert hätte.

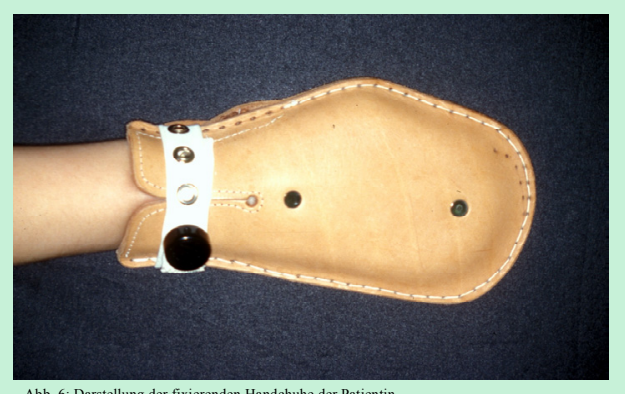

\section{Kasuistik 2}

31-jährige Patientin mit zunächst komplikationsloser Infundibulotomie und Turbinektomie. Präoperativ zeigte sich bereits eine Septumperforation unklarer Genese. Postoperativ kam es zu einer starken Angstreaktion, die nach einem psychiatrischen Konsil symptomatisch mit Sedativa behandelt wurde. Am 27. postoperativen Tag stellte sich die Patientin mit einen Hämoglobinwert von $50 \mathrm{~g} / 1$ vor und berichtete über rezidivierende Epistaxis von mehr als 30 Minuten Dauer. Die Transfusion zweie Erythrozytenkonzentrate war notwendig. Unter der weiteren Überwachung kam es zu einer erneuten, von der Patientin zunächst verschwiegenen Blutungsepisode mit einem Verlust von etwa $750 \mathrm{ml}$ Blut. Im erneuten psychiatrischen Konsil äußerte die Patientin, durch Manipulation in der Nase eine bewusst lange Blutungsphase zu provozieren und zu selbstverletzendem Verhalten zu neigen. Eine Therapie lehnte die Patientin ab. Ursächlich wird hier ebenfalls das Vorliegen einer Missbrauchs- und Mißhandlungssymptomatik in der Kindheit gesehen.

\section{Diskussion}

Im Rahmen verschiedener Erkrankungen ist über Inkorporationen von Fremdkörpern berichtet worden. Für den im Bereich der Hals-, Nasen- und Ohrenerkrunkungen tätigen Arzt sind aus der Literatur vor allem Falldarstellungen in Zusammenhang mit dem Transport von Drogen oder drogenassoziierten Verletzungen, zum Beispiel nach Injektionen im Halsbereich bekannt. [1- 2]. Im Bereich der psychiatrischen Erkrankungen stellt selbstverletzendes Verhalten ein häufiges unspezifisches Symptom [3] dar. Diese Störung muss sowohl vom Suizid, als auch von einer Simulation abgegrenzt werden. Es besteht jedoch eine erhöhte Neigung zum Suizid und zu suizidalen Ideen, vor allem bei mehrfacher Selbstverletzung [4]. Eine weitere Differenzierung erfolgt anhand der Form der Verletzung, wobei es sich sehr häufig um Ritzverletzungen der Haut oder Wundinfektionen handelt, und anhand der Häufigkeit und des Schweregrads der Verletzungen [3]. In der Pathogenese spielen verschiedene Faktoren in der kindlichen Entwicklung eine Rolle: Gewalterfahrungen, körperliche Misshandlung, sexueller Missbrauch, Verlust enger Bezugspersonen [3-4]. Bei den betroffenen Patienten finden sich häufig Impulskontrollstörungen, Somatisierungsmuster, emotionale Instabilität, dissoziales Verhalten, gesteigerte Fremd- und Eigenaggression, depressive Verstimmungen, Ängstlichkeit und zwanghaftes Verhalten, eine Borderline-Symptomatik, sowie Körperschemastörungen [3-4]. Überdurchschnittlich häufig findet sich auch eine Intelligenzminderung [5]. Auslöser für eine akute Verletzung sind häufig Spannungszustände der Betroffenen. Eine Affektbewältigung ist nicht möglich. Als Höhepunkt dieser Reaktion kommt es zur Selbstverletzung, welche ein Gefühl von Wohlbefinden und Erwachen hinterlässt. Innerhalb eines kurzen Zeitraumes kehrt sich dies um, was einen erneuten Spannungszustand erzeugt [4]. Die Behandlung der Selbstverletzung dient lediglich der akuten Intervention und sollte im Rahmen einer sehr langfristigen Therapie eingebettet sein, da eine Sedierung und Fixierung des Patienten die momentane Selbstverletzungshäufigkeit vermindern könnte, seine Lage langfristig jedoch verschlechtern würde.

\section{Fazit:}

Bei Vorliegen einer auffälligen Anamnese, fremdanamnestischen Berichten oder dem Vorhandensein auffälliger Merkmale wie Schnittmarken an den Unterarmen sollte die Möglichkeit selbstverletzenden Verhaltens auch bei unklaren Befunden im Kopf-Hals-Bereich in Betracht gezogen werden. Auffällig sind vor allem Inkorporationen und Manipulationen, nicht nur in der Nase, sondern zum Beispiel auch an den Ohren. Eine offensichtliche Gefahr ist der Progress von selbstschädigendem Verhalten bis zur Suizidalität. Bekannt ist aber auch, dass durch heimliche selbstmanipulierte Krankheitssymptome Untersuchungen und Behandlungen bis hin zu iatrogenen Schädigungen provoziert werden können, letzteres dann unter dem Bild der artifiziellen Störung. In beiden Fällen ist es erforderlich, einen Psychiater hinzuzuziehen. 